\title{
Predicting the requirement for renal replacement therapy in intensive care patients with sepsis
}

\author{
Axel Nierhaus ${ }^{1 *}$, Frank Bloos ${ }^{2,3}$, Darius Cameron Wilson ${ }^{4}$, Gunnar Elke ${ }^{5}$, Patrick Meybohm ${ }^{6}$ and the SepNet Critical \\ Care Trials Group
}

Sepsis is one of the most frequent causes of acute kidney injury (AKI) in critically ill patients, with initial organ impairment often followed by dysfunction in other systems [1]. Renal dysfunction may therefore represent one facet in the evolution towards multiple organ dysfunction syndrome (MODS) or, alternatively, may be indicative of system-wide endothelial damage caused by hyperinflammation and a positive fluid balance. Whilst numerous biomarkers have been investigated to predict renal replacement therapy (RRT) requirement, including NGAL, TIMP-2 and IGFBP-7 [2], mid-regional proadrenomedullin (MR-proADM) may also be of interest due to its involvement in capillary leakage, endothelial dysfunction and the initial stages of multiple organ failure development [3, 4].

In a secondary analysis of 1089 severe sepsis and septic shock patients enrolled in the SISPCT trial [5], RRT was initiated in $322(29.9 \%)$ patients within the first 21 days of treatment, including 178 (55.5\%; $52.2 \%$ mortality) patients at baseline and 118 (36.6\%; $55.1 \%$ mortality) additional patients between days 1-7. Continuous veno-venous haemodialysis (CVVHD: $N=88 ; 49.4 \%$ ) and haemodiafiltration (CVVHDF: $N=54 ; 30.3 \%$ ) were the most common modes of RRT at baseline.

Biomarker (PCT, MR-proADM, CRP and lactate) and standard clinical and laboratory parameters (creatinine, urea and 24-h urine output) were subsequently compared to identify RRT requirement at baseline (day 0), and predict requirement between days 1 and 7 in patients where no RRT was previously initiated. AUROC and logistic regression analysis found that urine output, MR-proADM and creatinine performed similarly in identifying RRT requirement at baseline, whereas MR-proADM more accurately predicted requirement between days 1 and 7 (Fig. 1). Previously established [3] MR-proADM cut-offs for predicting 28-day mortality found that increasing (e.g. moderate to high: $N=19$; 47.5\%; OR [95\% CI]: 67.6 [18.5 - 247.2]) or continuously elevated $(N=35 ; \quad 64.8 \% ; \quad$ OR $[95 \% \quad \mathrm{CI}]: \quad 137.5$ [38.7 - 489.1]) concentrations over the first $24 \mathrm{~h}$ in patients where no RRT was initiated at baseline resulted in a high likelihood of subsequent RRT requirement. Conversely, few cases of RRT over the first 21 days of ICU therapy were initiated in patients with continuously low $(N=3 ; 1.3 \%)$ or decreasing (moderate to low: $N=1$; 1.3\%) MR-proADM concentrations.

Results suggest that increasing or continuously elevated MR-proADM concentrations, indicative of increased capillary leak, may be a useful predictor of RRT requirement during ICU therapy. Further studies are required to investigate the relationship between MR-proADM, positive fluid balance and renal replacement therapy in critically ill patients with sepsis.

\footnotetext{
*Correspondence: nierhaus@uke.de

${ }^{1}$ Department of Intensive Care Medicine, University Hospital

Hamburg-Eppendorf, Hamburg, Germany

Full list of author information is available at the end of the article
} 

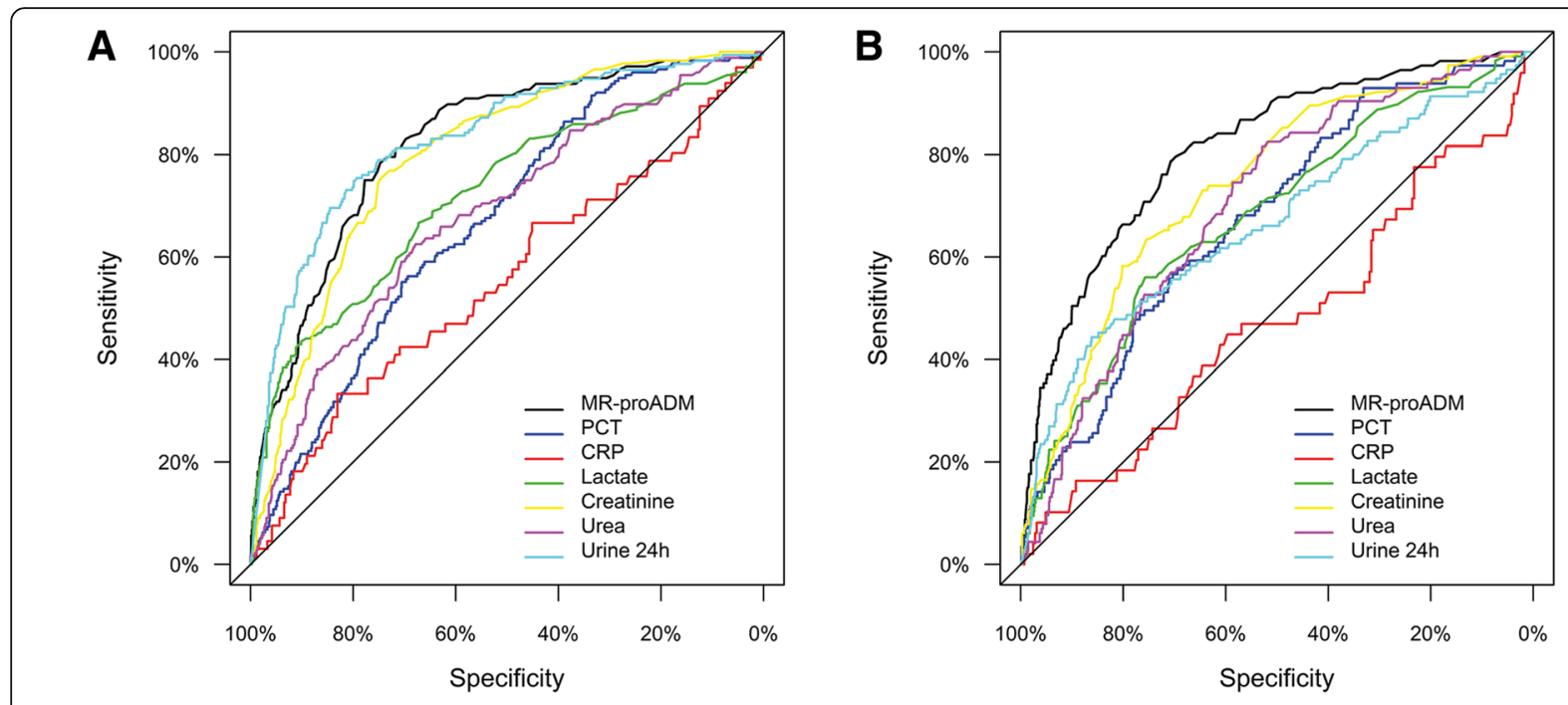

\begin{tabular}{|c|c|c|c|c|c|c|c|c|c|c|}
\hline & & & & & Un & ariate logistic rę & ssion & $\mathrm{Mul}$ & ariate logistic re & ession \\
\hline & $\begin{array}{l}\text { Biomarker or } \\
\text { lab parameter }\end{array}$ & $\begin{array}{l}\text { Patients } \\
\text { (N) }\end{array}$ & $\begin{array}{l}\text { RRT } \\
\text { (N) }\end{array}$ & AUROC & $\operatorname{LR} x^{2}$ & $\begin{array}{c}\text { OR } \\
\text { IQR }[95 \% \mathrm{Cl}]\end{array}$ & $\mathrm{p}$-value & $\operatorname{LR} \chi^{2}$ & $\begin{array}{c}\text { OR } \\
\text { IQR }[95 \% \mathrm{Cl}]\end{array}$ & $p$-value \\
\hline \multirow{7}{*}{ 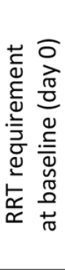 } & MR-proADM & 1030 & 176 & $0.82[0.79-0.86]$ & 202.9 & $8.7[6.1-12.5]$ & $<0.001$ & 239.4 & $8.0[5.5-11.7]$ & $<0.001$ \\
\hline & PCT & 1031 & 176 & $0.67[0.63-0.71]$ & 54.3 & $2.7[2.1-3.6]$ & $<0.001$ & 125.3 & $2.7[2.0-3.7]$ & $<0.001$ \\
\hline & CRP & 403 & 66 & $0.55[0.47-0.64]$ & 2.6 & $0.8[0.7-1.0]$ & 0.107 & 22.7 & $0.8[0.6-1.0]$ & $<0.001$ \\
\hline & Lactate & 1051 & 177 & $0.72[0.68-0.77]$ & 107.1 & $2.9[2.3-3.5]$ & $<0.001$ & 176.7 & $3.0[2.4-3.7]$ & $<0.001$ \\
\hline & Creatinine & 1058 & 177 & $0.80[0.76-0.83]$ & 163.4 & $5.0[3.8-6.7]$ & $<0.001$ & 192.8 & $4.4[3.2-5.8]$ & $<0.001$ \\
\hline & Urea & 1050 & 176 & $0.68[0.64-0.73]$ & 60.7 & $2.6[2.0-3.3]$ & $<0.001$ & 122.5 & $2.3[1.8-3.1]$ & $<0.001$ \\
\hline & Urine output & 1031 & 171 & $0.83[0.80-0.87]$ & 189.7 & $2.2[1.9-2.4]$ & $<0.001$ & 229.8 & $2.1[1.9-2.4]$ & $<0.001$ \\
\hline \multirow{7}{*}{ 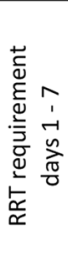 } & MR-proADM & 854 & 113 & $0.81[0.77-0.85]$ & 131.8 & $9.3[5.9-14.6]$ & $<0.001$ & 136.9 & $8.8[5.5-14.0]$ & $<0.001$ \\
\hline & PCT & 855 & 113 & $0.68[0.63-0.73]$ & 40.4 & $2.9[2.0-4.0]$ & $<0.001$ & 65.3 & $3.0[2.1-4.3]$ & $<0.001$ \\
\hline & CRP & 337 & 49 & $0.53[0.43-0.62]$ & 0.0 & $1.0[0.7-1.3]$ & 0.872 & 7.8 & $1.0[0.7-1.4]$ & 0.101 \\
\hline & Lactate & 874 & 116 & $0.68[0.63-0.73]$ & 39.8 & $2.3[1.8-3.0]$ & $<0.001$ & 56.4 & $2.2[1.7-2.9]$ & $<0.001$ \\
\hline & Creatinine & 881 & 115 & $0.74[0.69-0.79]$ & 75.9 & $3.7[2.7-5.1]$ & $<0.001$ & 92.6 & $3.7[2.7-5.0]$ & $<0.001$ \\
\hline & Urea & 874 & 114 & $0.70[0.65-0.75]$ & 45.6 & $2.7[2.0-3.7]$ & $<0.001$ & 61.2 & $2.6[1.9-3.6]$ & $<0.001$ \\
\hline & Urine output & 860 & 115 & $0.67[0.61-0.73]$ & 38.7 & $1.7[1.4-1.9]$ & $<0.001$ & 55.4 & $1.6[1.4-1.9]$ & $<0.001$ \\
\hline
\end{tabular}

Fig. 1 Identification of patients requiring renal replacement therapy (RRT) upon sepsis diagnosis and over the first week of ICU treatment. Logistic regression and AUROC analysis for the requirement of RRT in all patients at baseline (a) and during the first 7 days of ICU therapy in patients with no prior RRT (b). Multivariate Cox regression analysis was corrected for age and the presence of comorbidities. AUROC area under the receiver operating characteristic curve, Cl confidence interval, CRP C-reactive protein, IQR interquartile range, $L R$ likelihood ratio, MR-proADM mid-regional proadrenomedullin, $N$ number, $O R$ odds ratio, $P C T$ procalcitonin, $R R T$ renal replacement therapy

\section{Abbreviations}

AKI: Acute kidney injury; AUROC: Area under the receiver operating characteristic curve; Cl: Confidence interval; CRP: C-reactive protein; CWHD: Continuous veno-venous haemodialysis; CWHDF: Continuous venovenous haemodiafiltration; ICU: Intensive care unit; IGFBP-7: Insulin-like growth factor-binding protein 7; MODS: Multiple organ dysfunction syndrome; MR-proADM: Mid-regional proadrenomedullin; N: Number; NGAL: Neutrophil gelatinase-associated lipocalin; OR: Odds ratio; PCT: Procalcitonin; RRT: Renal replacement therapy; SISPCT: Placebocontrolled trial of sodium selenite and procalcitonin guided antimicrobial therapy in severe sepsis; TIMP-2: Tissue inhibitor of metalloproteinases 2

\section{Funding}

The SISPCT trial infrastructure was partially funded by grant $01 \mathrm{KI} 0106$ from the German Federal Ministry of Education and Research. Biosyn (Germany) and Thermo Fisher (Germany) provided study medication and financial support via unrestricted grants. For the purpose of this letter, no specific funding was received. Neither for the original SISPCT trial nor this letter did the funding organizations have any role in the design and conduct of the trial; collection, management, analysis, and interpretation of the data; preparation, review, or approval of the manuscript and decision to submit the manuscript for publication.

\section{Availability of data and materials}

The datasets used and/or analyzed during the present study are available from the corresponding author on reasonable request.

\section{Authors' contributions}

AN was the primary author and editor of the manuscript. FB was the principal investigator for the SISPCT trial. AN, FB, DCW, GE and PM collected the study data and contributed to the evaluation and interpretation of data as well as the writing and editing of the manuscript. AN, FB, DCW, GE and PM performed the statistical analysis of data. All authors critically reviewed and approved the final manuscript. 


\section{Ethics approval and consent to participate}

The study protocol of the SISPCT trial was approved by the ethics board of Jena University Hospital (internal file number 2242-03/08). Written informed consent was obtained from all patients or their legal representatives.

\section{Consent for publication}

No individual participant data are reported that would require consent to publish from the participant (or legal parent or guardian for children).

\section{Competing interests}

All authors have provided information on potential conflicts of interests directly or indirectly related to the work submitted in the journal's disclosure forms. AN reported receiving lecture honoraria from Thermo Fisher Scientific. FB reported receiving lecture honoraria from biosyn, Gilead and CSL Behring and public funding for the SISPCT trial to his department by the German Federal Ministry of Education and Research, as well as unrestricted research grants for the SISPCT trial by biosyn and Thermo Fisher Scientific. DCW is an employee of BRAHMS GmbH. The authors declare that they have no competing interests.

\section{Publisher's Note}

Springer Nature remains neutral with regard to jurisdictional claims in published maps and institutional affiliations.

\section{Author details}

Department of Intensive Care Medicine, University Hospital Hamburg-Eppendorf, Hamburg, Germany. ${ }^{2}$ Department of Anesthesiology and Intensive Care Medicine, Jena University Hospital, Am Klinikum 1, 07747 Jena, Germany. ${ }^{3}$ Center for Sepsis Control \& Care (CSCC), Jena University Hospital, Am Klinikum 1, 07747 Jena, Germany. ${ }^{4}$ B.R.A.H.M.S GmbH, Hennigsdorf, Neuendorfstr. 25, 16761 Hennigsdorf, Germany. ${ }^{5}$ Department of Anaesthesiology and Intensive Care Medicine, University Medical Center Schleswig-Holstein, Campus Kiel, Arnold-Heller-Str. 3 Haus 12, 24105 Kiel, Germany. ${ }^{6}$ Department of Anaesthesiology, Intensive Care Medicine and Pain Therapy, University Hospital Frankfurt, Theodor-Stern-Kai 7, 60590 Frankfurt am Main, Germany.

Received: 9 July 2018 Accepted: 25 July 2018

Published online: 20 August 2018

\section{References}

1. Ziesmann MT, Marshall JC. Multiple organ dysfunction: the defining syndrome of sepsis. Surg Infect. 2018;19(2):184-90.

2. Klein SJ, Brandtner AK, Lehner GF, et al. Biomarkers for prediction of renal replacement therapy in acute kidney injury: a systematic review and metaanalysis. Intensive Care Med. 2018;44(3):323-36.

3. Elke G, Bloos F, Wilson DC, et al. The use of mid-regional proadrenomedullin to identify disease severity and treatment response to sepsis--a secondary analysis of a large randomised controlled trial. Crit Care. 2018:22(1):79.

4. Elke G, Bloos F, Wilson DC, Meybohm P. Identification of developing multiple organ failure in sepsis patients with low or moderate SOFA scores. Crit Care. 2018:22(1):147.

5. Bloos F, Trips E, Nierhaus A. Effect of sodium selenite administration and procalcitonin-guided therapy on mortality in patients with severe sepsis or septic shock: a randomized clinical trial. JAMA Intern Med. 2016;176(9):1266-76. 